\title{
Erratum to: Scenario-Based Inundation Analysis of Metro System in Urban Area of Shanghai
}

Hai-Min Lyu, Shui-Long Shen, and Arul Arulrajah

\author{
Erratum to: \\ Chapter "Investigation of Ground Displacement Induced \\ by Hydraulic Jetting Using Smoothed Particle \\ Hydrodynamics" in: X. Shi et al. (Eds.): Proceedings \\ of GeoShanghai 2018 International Conference: Transportation \\ Geotechnics and Pavement Engineering, \\ https://doi.org/10.1007/978-981-13-0011-0_2
}

An error in the production process unfortunately led to publication of chapter 2 prematurely, before incorporation of the final corrections. The version supplied here has been corrected and approved by the authors.

The updated online version of this chapter can be found at https://doi.org/10.1007/978-981-13-0011-0_2 